The ASTROPHYSICAL JOURNAL SUPPLEMENT SERIES, 90:553-559, 1994 February

(c) 1994. The American Astronomical Society. All rights reserved. Printed in U.S.A.

\title{
DRIFT ACCELERATION AT INTERPLANETARY SHOCKS
}

\author{
G. ERDös \\ KFKI Research Institute for Particle and Nuclear Physics, H-1525 Budapest, POB 49, Hungary \\ AND \\ A. BALOGH \\ Space and Atmospheric Physics Group, The Blackett Laboratory, Imperial College, London SW7 2BZ, UK \\ Received 1993 February 26; accepted 1993 June 9
}

\begin{abstract}
Scatter-free acceleration of energetic particles by quasi-perpendicular interplanetary shocks is investigated. A brief review is given on the predictions of the gradient drift acceleration model concerning the energy, time, and angular dependence of the particle flux caused by a single shock encounter interaction. The angular distribution of ions in the energy range $35 \mathrm{keV}$ to $1 \mathrm{MeV}$ has been determined by the low-energy ion spectrometer aboard the ISEE 3 spacecraft at several shock associated events. Reflections of particles from the shock were clearly identifiable by the loss cone in the upstream pitch angle distributions. The measurements were compared to the predictions of the gradient drift acceleration model, showing a qualitative agreement in many respects. However, bidirectional distributions observed at nearly perpendicular shocks cannot be explained in the framework of the single shock encounter mechanism. It is suggested that multiple intersections of the field lines with the surface of the shock, forming magnetic traps on the upstream side, are responsible for the observed bidirectional distributions. Results obtained from numerical test particle simulations are discussed and compared to observations. A qualitative agreement between model calculations and measurements confirms that the energetic particles are trapped and accelerated, due to special field line topology, on the upstream side of the shock. It is also argued that the collapse of the trap by the convection of the field lines through the shock is accompanied by a considerable increase of the particle flux, which may be responsible for the shock spikes.
\end{abstract}

Subject headings: acceleration of particles - interplanetary medium

\section{INTRODUCTION}

Numerous observations carried out by spacecraft have shown that the flux of energetic particles often increases in close proximity to the passage of fast mode interplanetary shocks (for an early review, see Armstrong et al. 1977). The most spectacular phenomenon is the so-called "shock spike" event, observed especially at quasi-perpendicular shocks (Sarris \& Van Allen 1974; Sarris et al. 1976; Sarris \& Krimigis 1985). The spatial extent of these events is always very small, often comparable to the gyroradius of the particles. The increase of the flux is always closely connected with the shock passage; this implies that the shock is responsible for the accelerating the particles. It has been argued that diffusion processes may not play an important role in the acceleration mechanism (e.g., Krimigis 1987) as the spatial scale of the flux increase is probably much less than any scale length associated with diffusive propagation. Another important feature of the shock spike events, recognized very early on, is that the anisotropy of particles is usually quite large (Armstrong et al. 1970; Singer \& Montgomery 1971; Balogh \& Erdös 1983; Sanderson et al. 1985). A large deviation from the isotropy suggests, again, an essentially scatter-free propagation of particles during the acceleration process.

If scattering is unimportant, it is usually possible to find a suitable frame of reference in which the magnetic field can be regarded as stationary in time. It follows from the curl $E+$ $(1 / c)(\partial B / \partial t)=0$ induction law that in such a frame the elec- tric field should be curl-free. (This simple fact is sometimes not taken into account in numerical trajectory simulations.) The existence of an electric potential means that the energy gain (or loss) of a charged particle is simply related to the change of its coordinates. In a tenuous, collisionless plasma, where the electric field is usually perpendicular to the magnetic field (as in the case of an electric field induced by the plasma flow), the ultimate reason for the acceleration is therefore the cross-field motion of particles. This simple argument justifies the use of the terms "scatter-free acceleration," or "drift acceleration" almost as synonyms, although we have to be aware of some distinctive features behind these names.

The drift acceleration of energetic particles has been investigated in many publications over the past two decades (a selection of review papers on this topic includes Toptyghin 1980; Drury 1983; Armstrong, Pesses, \& Decker 1985, Forman \& Webb 1985; Decker 1987; Wenzel 1989). We do not intend to give here a comprehensive review of the observations and theoretical models associated with the shock drift process. The next section of this paper deals with the originally proposed form of the process in which, using a simplified geometrical setup, the particles encounter the shock only once. In that and the following sections, we propose to discuss some selected topics we consider interesting and important, with particular emphasis given to those aspects we find relatively new and controversial, or those quoted frequently in a potentially misleading manner.

The third section lists the observational evidence, in particular the bidirectional (pitch angle) distributions measured up- 
stream of quasi-perpendicular shocks, which cannot be explained by the single shock encounter process. Multiple crossing of field lines with the shock, forming magnetic traps on the upstream side are proposed to explain the observations. The comparison of observations with model predictions are discussed, together with the implications of the trap model on the effectiveness of drift acceleration which is enhanced, due to multiple shock encounters of particles.

\section{SINGLE SHOCK ENCOUNTER}

\subsection{The grad B Drift Model}

In an approximation which neglects scattering, we may consider the trajectory of energetic particles as travelling through an electromagnetic field averaged over large spatial scales (compared to their gyroradius). However, the smoothing of the magnetic field is not justified at the shock itself, where the physical quantities usually change in a distance shorter than the gyroradius of the particles. In the simplest form of the drift acceleration model, the magnetic field vector (together with the plasma flow vector) is taken to be homogeneous and stationary on both sides of a plane representing the surface of the shock. Both the magnetic field and solar wind flow vectors on the two sides of the shock are related through the Rankine-Hugoniot equations (Tidman \& Krall 1971). It is customary to treat the energetic particles as test particles: it is assumed that their contribution to the physical quantities describing the state of the plasma on the two sides of the shock, in particular to the plasma pressure, can be neglected.

The geometrical setup in what is usually called the shock rest frame, but more specifically is the normal incidence frame (in which the shock is at rest) is shown in Figure 1, together with the trajectories of particles representing three classes of shock interactions. These are

1. Transmission from upstream to downstream;

2. Reflection on the upstream side;

3. Transmission from downstream to upstream.

The reason for the acceleration is the grad $B$ drift of the particles, which happens to be parallel with the $\boldsymbol{E}=-\boldsymbol{u} \times \boldsymbol{B} / \mathrm{c}$ electric field, induced by the $\boldsymbol{u}$ plasma flow (Armstrong et al. 1977). We can understand this drift easily, without mathematical details, by considering the alternating step changes in the gyroradius $\left(r_{G}\right)$ of the particles during shock crossings. Clearly, the acceleration is restricted to a narrow domain $\left( \pm 2 r_{G}\right)$ at the shock. For the shake of completeness, we note that the curvature drift, also present at the shock front, decelerates the particles (Webb, Axford, \& Terasawa 1983). This process, however, is unimportant at quasi-perpendicular shocks, where the kink of the field line at the shock is small. For slow mode shocks, curvature drift results in energy gain, while gradient drift causes energy loss.

Many analytical and numerical calculations have explored the characteristic features of the gradient drift acceleration process. There are two important tools which can be exploited in the analytical calculations, based on adiabatic theory:

1. The approximate conservation of the $v_{\perp}^{2} / B$ first adiabatic invariant (magnetic moment) during the shock interaction process (Hudson 1965; Alekseyev \& Kropotkin 1970; Terasawa 1979).

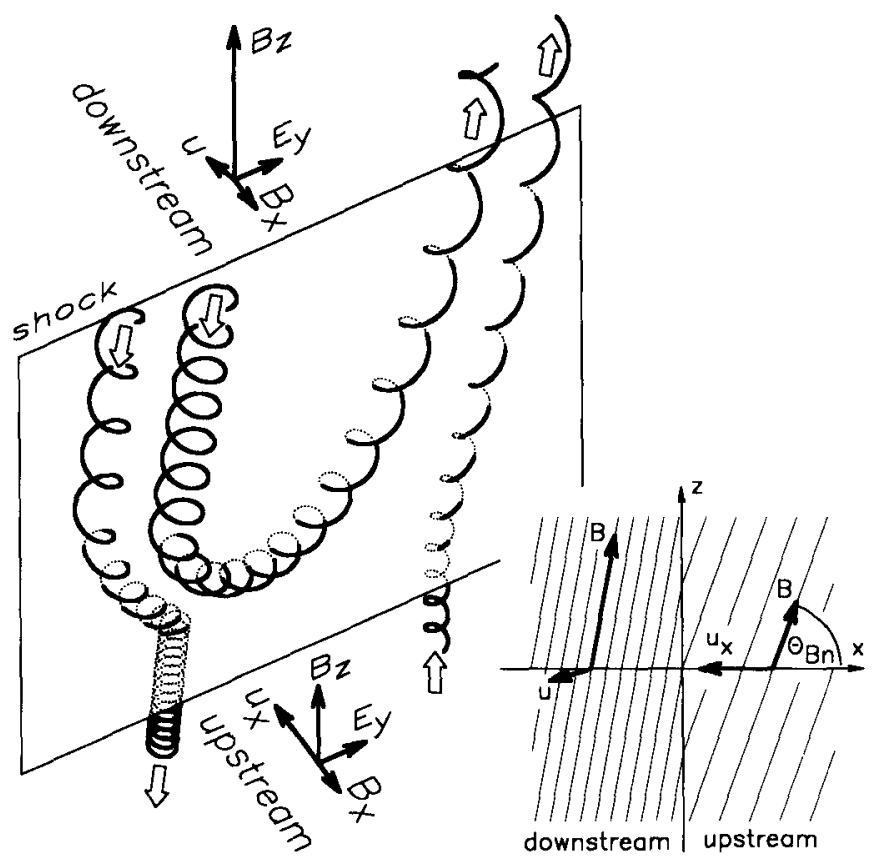

FIG. 1.- Magnetic field and plasma flow vectors in the normal incidence frame, and typical trajectories of energetic particles interacting with the shock.

2. The existence of a frame free of electric field (deHoffman \& Teller 1950).

The existence of the electric field-free frame, the deHoffman-Teller frame, follows directly from the sourceless Maxwell equations (from Faraday's law and from the divergencefree condition of $\boldsymbol{B}$ ), implying that the normal component of the magnetic field $\left(\boldsymbol{B}_{x}\right)$ and the electric field $\left(\boldsymbol{E}_{y}\right)$ are continuous across the shock (even if the shock has structure but only in the direction of the shock normal). The electric field can be transformed to vanish by a Lorentz transformation, using an appropriate velocity along the shock surface in the $z$ direction (for the notation used here, see Fig. 1). The speed of transformation, called also the effective shock velocity, is $w_{\text {shock }}=$ $u_{x} \tan \theta_{B n}$. We may generalize the meaning of the effective shock velocity as the transformation velocity from any frame of reference to the deHoffman-Teller frame. In particular, the effective shock velocity in the plasma frame is $w=u_{x} / \cos \theta_{B n}$, where $u_{x}$ now stands for the velocity of the shock (along the shock normal). The effective shock velocity (the velocity of the kink in the field line caused by the shock) strongly depends on the angle $\theta_{B n}$ between the magnetic field vector and the shock normal. Note that if $w$ exceeds the velocity of light, we cannot transform to the deHoffman-Teller frame (but we may transform to another frame where there is no normal component of the magnetic field). This case is highly unlikely at interplanetary shocks, since it would require a $\Theta_{B n}$ extremely close (of the order of an arcminute) to the perpendicular.

\subsection{Energy Dependence}

There are two (approximate) scaling symmetries which can be exploited in connection with the study of the energy depen- 
dence of the acceleration process. One is in the velocity space of particles. The most important parameter of the acceleration, measured in velocity units, is the effective shock velocity $w$, which should be compared to the particle velocity $v$. As the results of analytical calculations show, for quasi-perpendicular shocks, all physical quantities, including the effectiveness of acceleration, depend, in practice, on the particle energy through the dimensionless parameter $v / w$.

Another scaling symmetry can be established in the coordinate space, by neglecting any spatial structure other than the discontinuity at the shock. It can be shown that the trajectory of particles is similar (in the strict mathematical sense), if the velocity vector (as seen from the deHoffman-Teller frame) at the shock has the same direction but different magnitude ( $\mathrm{Ba}$ logh \& Erdös 1981). The scale factor of the trajectories is simply proportional to the particle velocity. Since the drift is the only way to gain energy (in the shock-rest frame), the energy gain is proportional to the particle velocity expressed in the deHoffman-Teller frame. The only complication is the change of the particle velocity imposed by the Lorentz transformation from the shock-rest frame to the deHoffman-Teller frame. In the high-energy limit, where the transformation has negligible effect, the energy change during the shock interaction $(\Delta E)$, normalized to the postinteraction energy $(E)$ is therefore $(\Delta E /$ $E) \sim(1 / \sqrt{E})$. If, however, the particle velocity is close to the effective shock velocity $w$, the transformation has a significant effect. In fact, there is a low-energy cutoff (at $v=w$ ) for the acceleration of particles observed upstream of the shock, simply because a particle reflected from the shock cannot move slower than the kink in the field line.

Figure 2 gives the results of an analytical calculation by Decker (1983), showing the predicted post interaction spectrum of particles, both upstream and downstream, supposing a power-law spectrum for the ambient population. According to the discussion of energy dependence above, the energy scale is given in units of $(v / w)^{2}$. The acceleration of particles is restricted to a relatively small energy interval, especially for those observed upstream. We may note in Figure 2 the decreasing effectiveness of the acceleration at higher energies, and also the cutoff in the upstream distribution at low energies. When interpreting particle observations at quasi-perpendicular shocks, we can always increase the effectiveness of the acceleration at higher energies, without contradicting the magnetic field measurements by supposing a $\theta_{B n}$ angle very close to $90^{\circ}$, since the direction of the shock normal is not known to high accuracy. However, we should bear in mind that a hypothetical large effective shock velocity would exclude the reflection of lower energy particles from the shock, which may contradict the particle observations.

\subsection{Time Dependence of the Flux}

The drift of particles, and therefore their acceleration, takes place close to the shock (in $\pm 2 r_{G}$ distance from the surface). This, however, does not necessarily mean that the energetic particles are accumulated close to the shock. Liouville's theorem applies in this case stating that the phase space density $f(\boldsymbol{x}, \boldsymbol{p}, t)$ is conserved along particle trajectories. The omnidirectional flux of energetic particles, a quantity measured as a

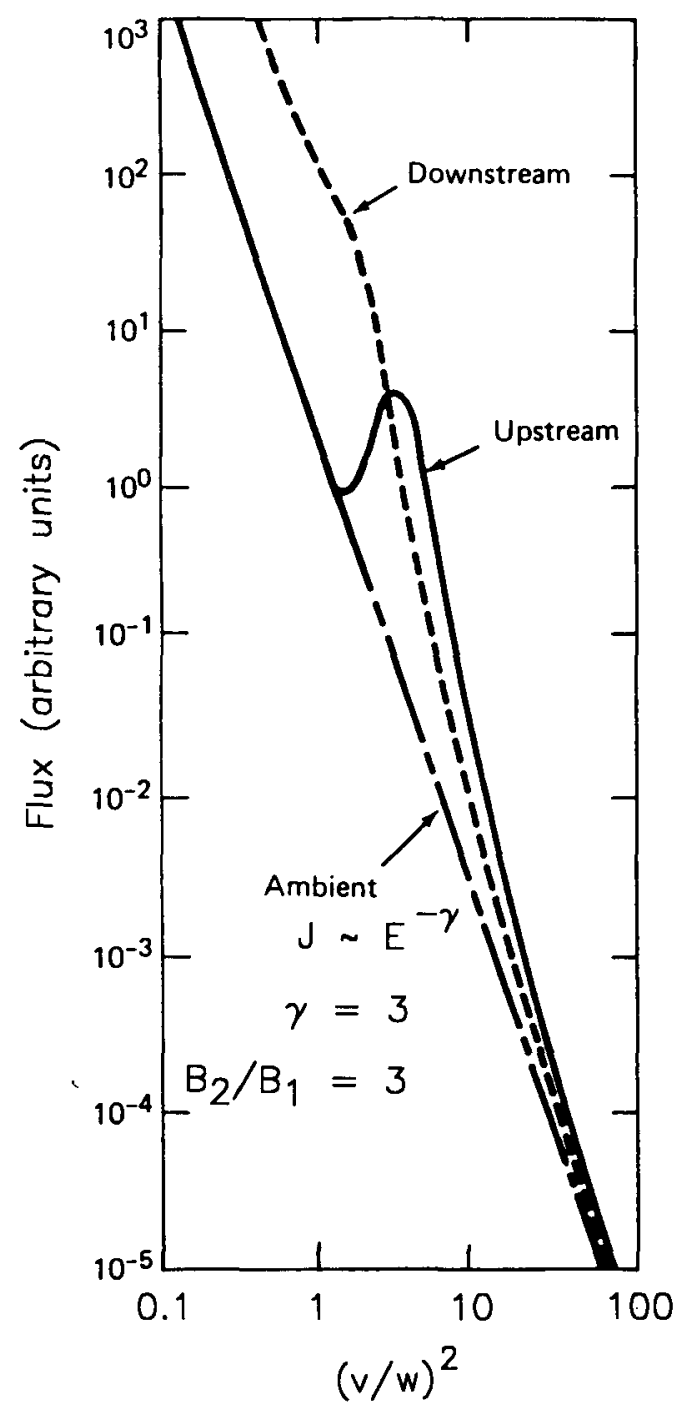

FIG. 2.-Predicted flux of particles, accelerated by a quasi-perpendicular shock (adapted from Decker 1983).

function of time in shock spike events is related to the phase space density by an integration over angles in momentum space:

$$
J(x, E)=\int p^{2} f(\boldsymbol{x}, \boldsymbol{p}, t) d \Omega_{p} .
$$

The flux $J$ is independent of time $t$ in the shock rest frame, and also independent of coordinates $y$ and $z$, according to our assumptions given in $\$ 2.1$ (the absolute value of the momentum is substituted by the energy). Thus, Liouville's theorem imposes a constraint on the energy spectrum, as a function of distance from the shock (or as a function of time as measured at the spacecraft). Since the flow of particles does not diverge, the omnidirectional flux should be the same outside of the acceleration region (i.e., beyond $\pm 2 r_{G}$ distance from the shock), on either side of the shock. Inside that region, a smooth transition from the upstream to the downstream flux 
level is expected, without a noticeable peak. Time reversed numerical trajectory calculations have confirmed the lack of shock accumulation (Erdös \& Balogh 1990). The problem of explaining shock spike events is not only the identification of an effective acceleration mechanism at the shock, but also the need to identify a loss, or confinement mechanism, which would lead to a decrease in the flux on a short spatial scale. Introducing time variation in the effectiveness of acceleration ( through the time variation of $\theta_{B n}$ ) may help but, in that case, we need to explain why the peak flux is usually observed close to the time when the shock passes through the spacecraft. Therefore the single encounter model, with those assumptions described in $\$ 2.1$ cannot really account for the characteristic features or even the existence of the shock spike.

\subsection{Anisotropy}

The fate of particles approaching the shock from the upstream side is different, depending on the pitch angle. Upstream particles with small pitch angle are transmitted downstream and, as a consequence of the adiabatic invariance, the pitch angle increases. If the pitch angle of the incident particle is large, then even an increase of the pitch angle to $90^{\circ}$ would not be adequate to maintain the invariance of the magnetic moment, therefore the particle is reflected from the shock.

It is frequently quoted that the downstream pitch angle distribution is a "pancake" distribution ( a distribution peaking at $90^{\circ}$ ), due to the increase of the pitch angle of the transmitted particles while, in the upstream region, there is a "field aligned flow" due to the increase of particle velocities parallel to the field line when reflected from the shock. If, however, we scrutinize the predictions of the gradient drift model, we may notice important differences (Fig. 3). Both the downstream and upstream distributions are discontinuous at the pitch angle separating the particles originating from the two sides of the shock. The peak intensity is also expected at that angle. We can understand this feature by inspecting the trajectory of particles ( see Fig. 1). Those particles which just escape downstream, or are just reflected upstream drift the longest distance along the shock front (and therefore gain the most energy).

The peak in the downstream distribution (Fig. 3, left side) does not occur naturally at $90^{\circ}$ pitch angle, although in the high-energy limit, when the acceleration is relatively small, the peak does occur close to $90^{\circ}$. The position of the peak intensity is expressed in the form of $\mu=w / v$, where $\mu$ is the cosine of the
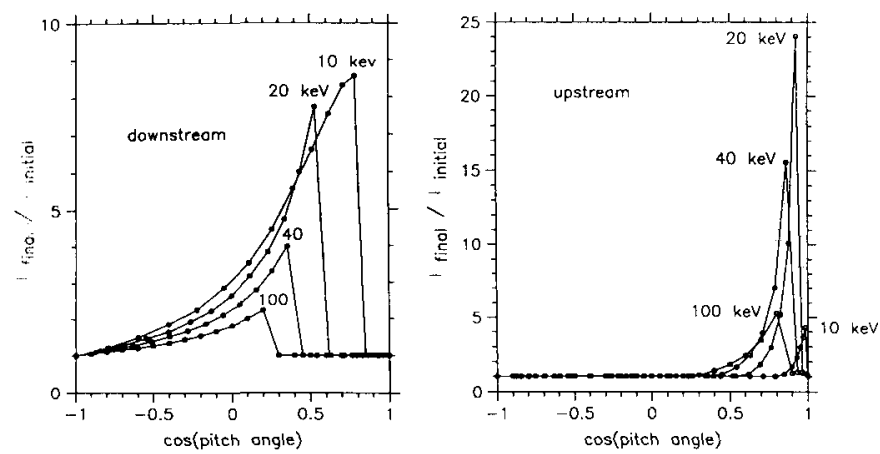

Fig. 3.-Numerically simulated pitch angle distributions of protons downstream (lefi) and upstream (right) of a quasi-perpendicular shock.

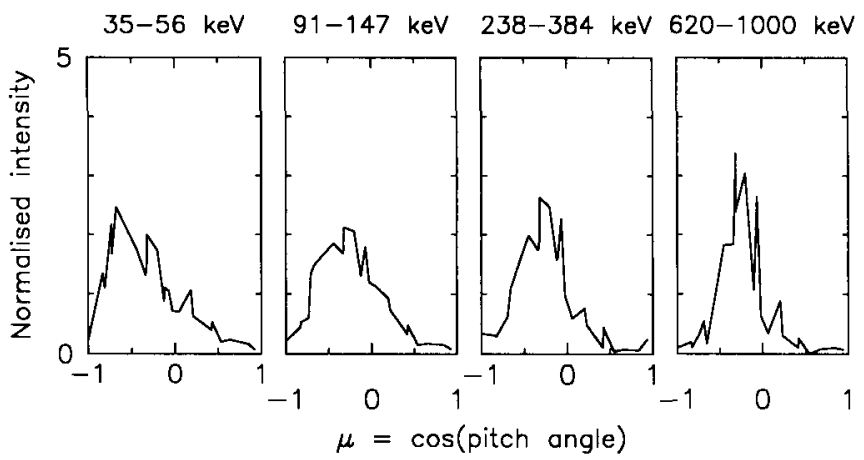

FiG. 4.-Pitch angle distributions of ions observed downstream of the interplanetary shock on 1979 November 30 .

pitch angle. The shift of the peak flux toward $\mu=0$ with increasing particle energy was observed by the low-energy ion spectrometer aboard ISEE 3 downstream of the 1979 November 30 shock, as shown in Figure 4 (Erdös \& Balogh 1990). By comparing the observation with the theoretical results presented in Figure 3, we have to bear in mind that the sense of the magnetic field along the field line was supposed to be opposite in the model from that observed in this shock associated event. ( This corresponds to a mirroring of the distribution with respect to $\mu=0$ ). A qualitative agreement between the model calculation and observations can thus be established except that the observations show no sign of less acceleration at high energy.

Turning to the more interesting upstream distribution (Fig. 3 , right side), a sharp peak is expected close to $\mu=1$ (or close to $\mu=-1$, if the magnetic field has opposite sense to that in the model calculations). The position of the peak changes little with particle energy. In the high-energy limit, the peak is at $\mu=$ $\left(1-B_{u} / B_{d}\right)^{1 / 2}$ (where $B_{d} / B_{u}$ is the magnetic compression ratio). Such a distribution is characteristic of the existence of a loss cone, due to the escape of particles downstream at small pitch angles. Loss cone distributions upstream of quasi-perpendicular interplanetary shocks have been observed in several events by the ISEE 3 spacecraft (Balogh \& Erdös 1985, 1991). Figure 5 shows the pitch angle distribution of $91-147 \mathrm{keV}$ ions observed just prior to the arrival of a shock on 1979 November

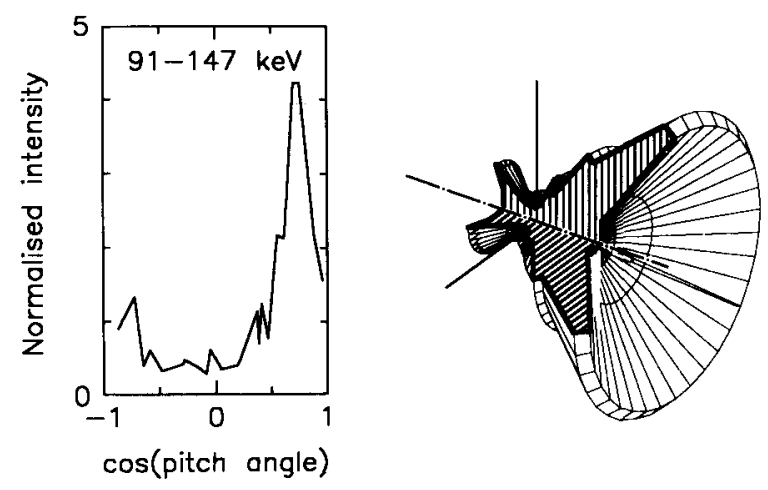

FIG. 5.-Pitch angle distribution of ions observed upstream of the interplanetary shock on November 1979 November 18. 
18. The depletion of particle flux at $\mu \approx 1$ is apparent, according to theoretical expectations (Fig. 3, right side).

Taking the magnetic compression ratio $B_{d} / B_{u}=2$ as a typical value, the half opening angle of the loss cone is as large as $45^{\circ}$. This means that, although the peak and the depletion in the flux are restricted to a very narrow domain in $\mu$, the loss cone could be seen, even with a telescope with a relatively coarse angular resolution. In order to illustrate this, the threedimensional reconstruction of the angular distribution measured by the ISEE 3 spacecraft is displayed on the right side of Figure 5. Our conclusion is that, according to the scatter-free model, not the field aligned flow but, rather the loss cone is the clear evidence for particle reflection at quasi-perpendicular shocks.

\section{MULTIPLE SHOCK ENCOUNTERS}

\subsection{Bidirectional Distributions}

Many characteristics predicted by the scatter-free model have been observed at quasi-perpendicular shocks. There is, however, a striking feature, which is not readily explainable in the framework of the original gradient drift model. Upstream of some shocks, especially at those with the closest to perpendicular shock normal to field line angle, the angular distribution observed is bidirectional, with loss cones in both directions along the field line (Balogh \& Erdös 1983, 1991; Erdös \& Balogh 1993). Such a distribution is displayed in Figure 6, measured by the low energy ion telescope aboard ISEE 3 just prior to the arrival of a quasi-perpendicular shock $\left(\Theta_{B n}=88^{\circ}\right)$ on 1978 December 25.

The time evolution of the pitch angle distribution of 91-147 $\mathrm{keV}$ ions through the shock on 1979 November 30 is shown in Figure 7. The measurements indicate that about 5 minutes prior to the shock arrival, the distribution became bidirectional and that this feature persisted until the shock passage. Again, loss cones in both directions along the field line were observed.

Bidirectional flow (but without loss cones) of protons as well as electrons were also observed by Potter (1981) upstream of quasi-perpendicular shocks. Figure 8 shows the angular distribution of $2 \mathrm{keV}$ electrons just prior to the arrival of a shock on 1979 July 26.


FIG. 6.-Pitch angle distribution of ions observed upstream of the interplanetary shock on 1978 December 25.



FIG. 7.-Time evolution of the pitch angle distribution of ions, associated with the shock event on 1979 November 30 .

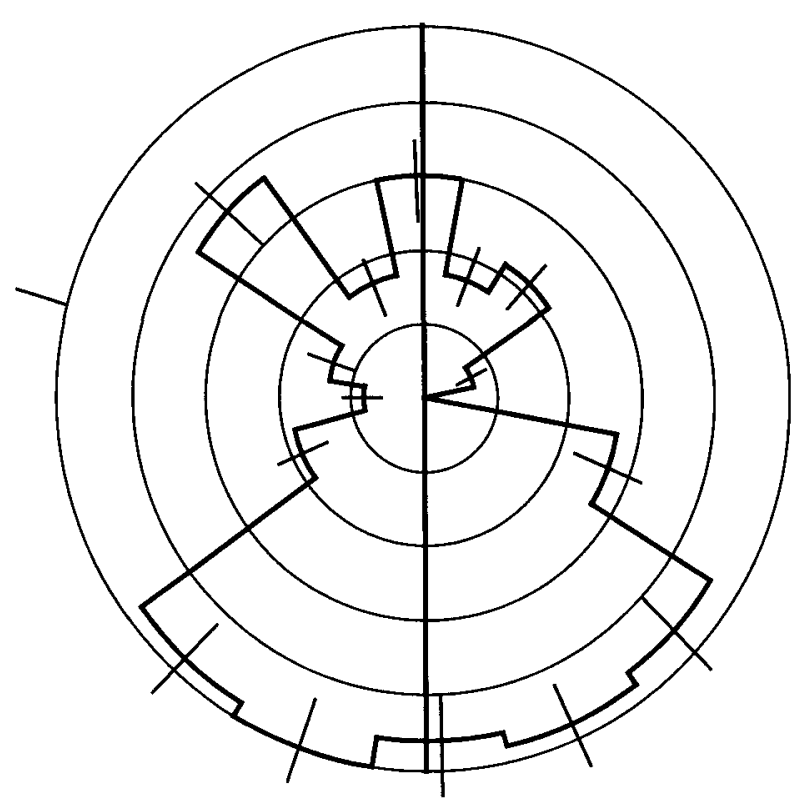

FIG. 8.-Angular distribution of 2-7 $\mathrm{keV}$ electrons in the ecliptic plane, observed upstream of the interplanetary shock on $1979 \mathrm{July} 26$. The vertical line is the projection of magnetic field vector in that plane (adapted from Potter 1981). 


\subsection{Trap Model}

As it was argued above, the presence of a loss cone in the angular distribution indicates the reflection of energetic particles from the shock. Since we have observed loss cones in both direction along the field line upstream of the shock, our explanation for the bidirectional distributions is that the field line at the observer intersects the shock both ways, forming a shortlived trap upstream of the shock. The topology of field line is the key feature, rather than the actual geometrical setup. Multiple shock crossings of field lines may happen either if the surface of shock is rippled (Decker 1990) or, if the field line is curved back to the shock (Balogh \& Erdös 1983, 1991; Gisler \& Lemons 1990; Decker 1993; Erdös \& Balogh 1993).

Time-reversed numerical integration of test particle trajectories have been performed to verify the hypothesis that the angular distribution of particles trapped upstream of quasiperpendicular shocks is bidirectional. The field line configuration used in the simulation is shown in Figure 9 together with the trajectory of a trapped particle. Pitch angle distributions, as a function of distance from the shock, calculated using the model, are given in Figure 10. We may note the remarkably good qualitative agreement between the results of the model calculations and the reported observations, especially those in Figure 7.

Beyond the explanation of bidirectional distributions, the trap model has some more general, interesting aspects as well. One is that the effectiveness of the acceleration is enhanced, due to multiple interaction (hence acceleration) with the shock. This could be an important factor especially at higher energies, where the smaller energy gain at each individual



Fig. 9.-Magnetic field lines in the trap model, and the trajectory of a 1 $\mathrm{MeV}$ proton.

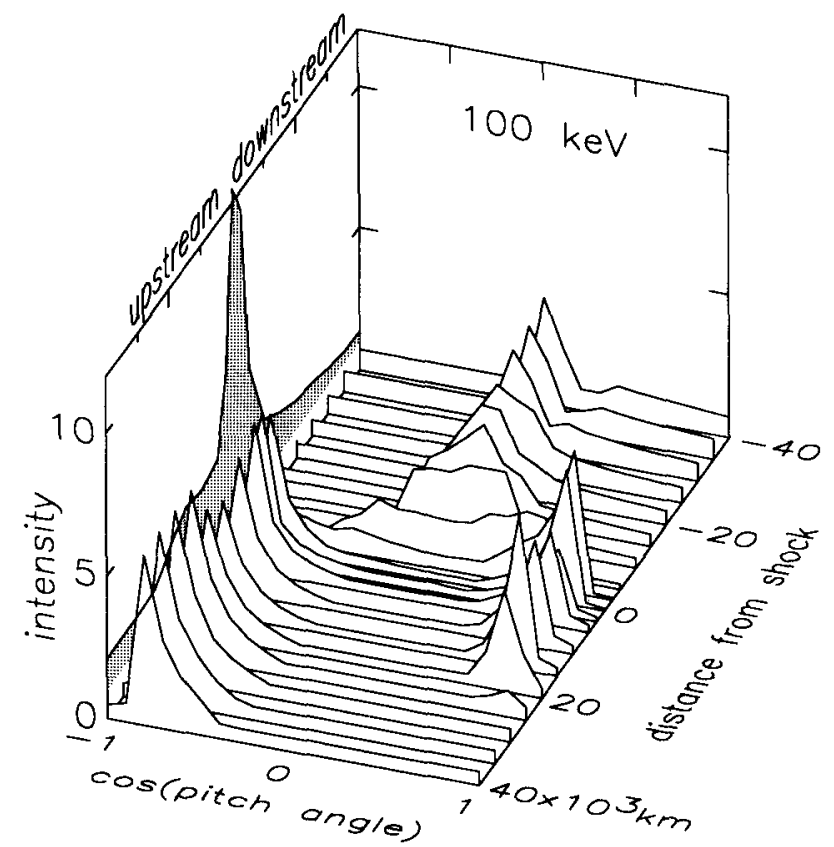

FIG. 10.-Numerically simulated pitch angle distributions of $100 \mathrm{keV}$ protons as a function of the distance from the shock.

shock reflection might be partly compensated by a greater number of shock encounters (Balogh \& Erdös 1983). This may extend the energy interval (and also the time) available for the acceleration considerably, as compared to the limitations of the single shock encounter process discussed in the previous section. Another interesting feature of the trap geometry is that, unlike for single shock encounters, the flux of particles, as seen by an observer traveling through the trap, could signifcantly vary in time. By averaging the predicted flux over pitch angles in the model calculations, the omnidirectional flux can be calculated as a function of the distance from the shock. We note in Figure 11 a sharp peak in the omnidirectional flux at

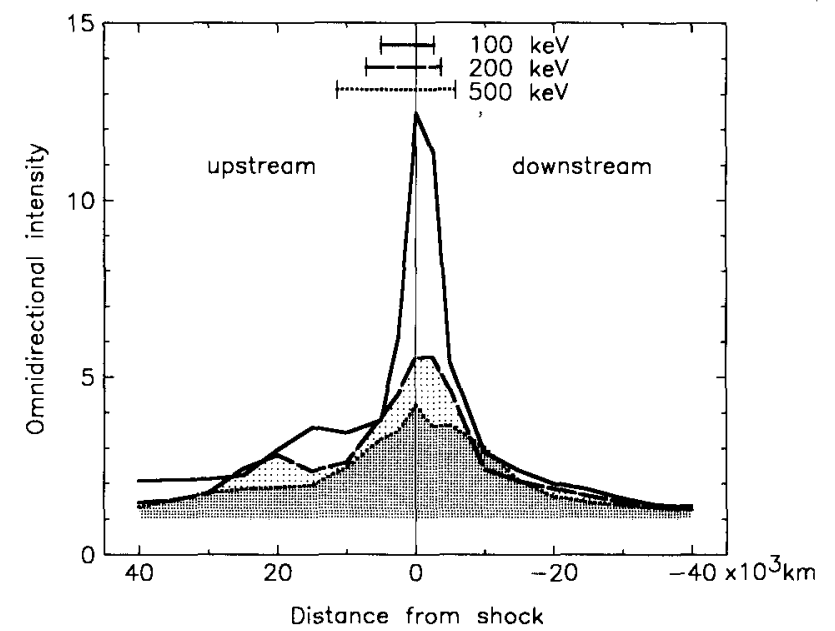

Fig. 11.-Numerical evaluation of the omnidirectional flux of 100 $\mathrm{keV}, 200 \mathrm{keV}$, and $500 \mathrm{keV}$ protons resulting from the trap model of shock acceleration. The upstream and downstream values of the gyroradii of the different energy particles are shown at the top of the figure to the left and the right of the shock front, respectively. 
the shock, on a spatial scale corresponding to the gyroradius of the particles. The enhanced flux is attributed to the collapse of the trap when the field line is convected downstream. Our suggestion is that the collapse of the trap is the explanation of shock spike events.

\section{CONCLUSIONS}

We have discussed the characteristic features of particle fluxes, expected from a single shock encounter drift acceleration model, which can be summarized as follows:

1. The acceleration of particles is restricted to a small energy interval, especially for those observed upstream of the shock.

2. According to theoretical considerations which assume steady state geometry, the particles are not accumulated close to the shock, in spite of the fact that they were accelerated in a very short distance $\left( \pm 2 r_{G}\right)$.

3. The downstream angular distribution is not expected to peak at $90^{\circ}$ pitch angle (pancake distribution), the position of the peak is shifted to smaller pitch angles with decreasing particle energy.

4. On the upstream side, not the field-aligned flow of the particles, but rather the loss cone, is the evidence for particle reflection from the shock, which is supported by ISEE 3 observations.
It was suggested that at nearly perpendicular shocks, the magnetic field lines may cross the shock front several times, forming traps on the upstream side and thus, providing an opportunity for multiple shock encounters by the particles. The most important predictions of the trap model are the following:

5. The upstream distribution of trapped particles is bidirectional, with loss cones in both ways along the field lines, as supported by observations.

6. The flux of particles through the trap is expected to change considerably, forming a narrow spike close to the shock.

In this paper we have neglected the scattering of particles by waves, simply because we wanted to explore what observational features could be attributed to a pure gradient drift acceleration model. In realistic situations, a more comprehensive treatment might be necessary. This can be achieved either by including fluctuations of the magnetic field in the test particle simulations (Decker \& Vlahos 1986; Decker 1993), or incorporating the drift term in the diffusive transport equation, as suggested by Jokipii (1982).

We wish to thank the referee for pointing out an error in the original manuscript and for helping us to clarify several other arguments.

\section{REFERENCES}

Alekseyev, I. I., \& Kropotkin, A. P. 1970, Geomagn. Aeron., 10, 755

Armstrong, T. P., Krimigis, S. M., \& Behannon, K. W. 1970, J. Geophys. Res., 75, 5980

Armstrong, T. P., Chen, G., Sarris, E. T., \& Krimigis, S. M. 1977, in Study of Traveling Interplanetary Phenomena, ed. M. A. Shea et al. (Dordrecht: Reidel ), 367

Armstrong, T. P., Pesses, M. E., \& Decker, R. B. 1985, in Collisionless Shocks in the Heliosphere: Reviews of Current Research, ed. B. T. Tsurutani \& R. G. Stone (Washington, AGU:), 271

Balogh, A., \& Erdös, G. 1981, Proc. 17th Internat. Cosmic Ray Conf., 3, 442

1983, Planet. Space Sci., 31, 1389

1985, Proc. 19th Internat. Cosmic Ray Conf., 4, 178

. 1991, J. Geophys. Res., 96, 15853

Decker, R. B. 1983, J. Geophys. Res., 88, 9959

1987, in Proc. Int. Conf. Collisionless Shocks, Balatonfüred, Hun-

gary, ed. K. Szegö (Budapest: Hung. Acad. Sci., KFKI), 224

1990, J. Geophys. Res., 95, 11993

1993, J. Geophys. Res., 98, 33

Decker, R. B., \& Vlahos, L. 1986, J. Geophys. Res., 91, 13349

deHoffman, F., \& Teller, E. 1950, Phys. Rev., 80, 692

Drury, L. O.'C. 1983, Rep. Progr. Phys., 46, 973

Erdös, G., \& Balogh, A. 1990, Planet. Space Sci., 38, 343
Erdös, G, \& Balogh, A. 1993, J. Geophys. Res., 98, 13253

Forman, M. A., \& Webb, G. M. 1985, in Collisionless Shocks in the Heliosphere: A Tutorial Review, ed. R. G. Stone \& B. T. Tsurutani (Washington: $A G U), 91$

Gisler, G., \& Lemons, D. 1990, J. Geophys. Res., 95, 14925

Hudson, P. D. 1965, MNRAS, 131, 23

Jokipii, J. R. 1982, ApJ, 255, 716

Krimigis, S. M. 1987, in Proc. Int. Conf. Collisionless Shocks, Balatonfüred, Hungary, ed. K. Szegö ( Budapest: Hung. Acad. Sci., KFKI), 3

Potter, D. W. 1981, J. Geophys. Res., 86, 11111

Sanderson, T. R., Reinhard, R., van Nes, P., \& Wenzel, K.-P. 1985, J. Geophys. Res., 90, 19

Sarris, E. T., \& Krimigis, S. M. 1985, ApJ, 298, 676

Sarris, E. T., \& Van Allen, J. A. 1974, J. Geophys. Res., 79, 4157

Sarris, E. T., Krimigis, S. M., \& Armstrong, T. P. 1976, Geophys. Res. Lett., 3, 133

Singer, S., \& Montgomery, M. D. 1971, J. Geophys. Res., 76, 6628

Terasawa, T. 1979, Planet. Space Sci., 27, 193

Tidman, D. A., \& Krall, N. A. 1971, Shock Waves in Collisionless Plasmas (New York: Wiley)

Toptyghin, I. N. 1980, Space Sci. Rev., 26, 157

Webb, G. M., Axford, W. I., \& Terasawa, T. 1983, ApJ, 270, 537

Wenzel, K.-P. 1989, Adv. Space Res., 9, No. 4, 179 